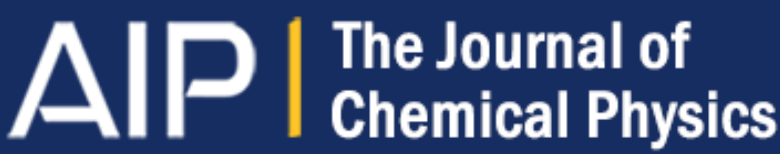

Theory of vibrational equilibria and pooling at solid-diatom interfaces

E. T. D. Boney and R. A. Marcus

Citation: The Journal of Chemical Physics 139, 124107 (2013); doi: 10.1063/1.4821991

View online: http://dx.doi.org/10.1063/1.4821991

View Table of Contents: http://scitation.aip.org/content/aip/journal/jcp/139/12?ver=pdfcov

Published by the AIP Publishing

Advertisement:

\section{AlP Re-register for Table of Content Alerts}




\title{
Theory of vibrational equilibria and pooling at solid-diatom interfaces
}

\author{
E. T. D. Boney and R. A. Marcus ${ }^{a)}$ \\ Noyes Laboratory of Chemical Physics, California Institute of Technology, Pasadena, \\ California 91125-7200, USA
}

(Received 16 July 2013; accepted 9 September 2013; published online 24 September 2013)

\begin{abstract}
In the present paper we provide a statistical theory for the vibrational pooling and fluorescence time dependence observed in infrared laser excitation of $\mathrm{CO}$ on an $\mathrm{NaCl}$ surface. The pooling is seen in experiment and in computer simulations. In the theory, we assume a rapid equilibration of the quanta in the substrate and minimize the free energy subject to the constraint at any time $t$ of a fixed number of vibrational quanta $N(t)$. At low incident intensity, the distribution is limited to one-quantum exchanges with the solid and the Debye frequency of the solid plays a key role in limiting the range of this one-quantum domain. The resulting inverted vibrational equilibrium population depends only on fundamental parameters of the oscillator $\left(\omega_{e}\right.$ and $\left.\omega_{e} \chi_{e}\right)$ and the surface $\left(\omega_{D}\right.$ and $\left.T\right)$. The relation to the Treanor gas phase treatment is discussed. Unlike the solid phase system, the gas phase system has no Debye-constraining maximum. (C) 2013 AIP Publishing LLC. [http://dx.doi.org/10.1063/1.4821991]
\end{abstract}

\section{INTRODUCTION}

Recently, the infrared absorption of $\mathrm{CO}$ on $\mathrm{NaCl}$ at low temperatures was calculated using Monte Carlo. ${ }^{1,2}$ In this paper, we describe such a statistical theory to explain two key effects: ${ }^{1-5}(1)$ an inversion of the population of $\mathrm{CO}$ vibrational states and (2) the origin of the single exponential overtone fluorescence decay, the many contributing second-order and first-order steps in the mechanism notwithstanding.

The present statistical form is of the same type as that derived by Treanor et al. ${ }^{6}$ for pooling of vibrational energy, except that since his treatment dealt with gases, he did not have a Debye cutoff. A comparison and possible extension of Treanor's results are given in Sec. IV.

A relative inverted peak in the vibrational population distribution is possible when there is a phonon bottleneck, e.g., when the average energy of the phonons emitted by a pooling step to reach a still higher vibrational state $n$ exceeds $\hbar \omega_{D}$, where $\omega_{D}$ is the Debye frequency of the solid. This situation is somewhat unusual, because it requires no low energy electronic, rotational, bending or vibrational transitions with which the high frequency stretch, in this case $\mathrm{CO}$, can decay in less than a large number of quanta, resulting in relaxation on the $m s$ timescale. ${ }^{10}$

In this work, we derive an expression for the approximate statistics and dynamics of single-phonon processes up to the first pooling maximum, recognizing that higher fluence results may lead to other, higher local maxima in $n$, a result we probe separately. ${ }^{3}$

\section{STATISTICAL TREATMENT OF VIBRATIONAL ENERGY DISTRIBUTION}

In the theory, we assume that after injection of infrared quanta, vibrational pooling and depooling lead to rapid equilibration among the vibrational states of the system at each

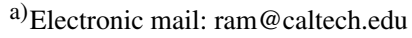

time $t$. Consistent with the available information on the individual rate constants for $\mathrm{CO}: \mathrm{NaCl}$, but applicable to monolayers on other surfaces if pooling occurs, we assume that the multi phonon relaxation by energy transfer to the solid is slower than the single-phonon-mediated pooling equilibration, so we treat the deactivation separately, as in Sec. III. The number of sites $M$ and the total number of quanta $N(t)$ in the system (the adsorbed $\mathrm{CO}$ ) can be expressed in terms of the occupation numbers $m_{n}$ of each site as

$$
\begin{gathered}
M=\sum_{n} m_{n}(t), \\
N(t)=\sum_{n} n m_{n}(t), \\
F(t)=E(t)-T S(t)=\sum_{n} m_{n}(t) \epsilon_{n}-k T \ln \frac{M !}{\prod_{n} m_{n}(t) !},
\end{gathered}
$$

where $E$ is the total vibrational energy at time t, $\epsilon_{n}$ is the energy of the $n$th vibrational state of an adsorbed molecule, $S(t)$ is the entropy of the adsorbate, $F(t)$ is the free energy, and $S=k \ln W$, where $W$ is the number of ways of distributing the $N$ quanta among the adsorbed molecules. For the purposes of simplicity, given the long timescales of the relaxation in question, we consider primarily an after laser excitation picture, where quanta are initially distributed according to the absorbed fluence in the calculations.

We minimize $F$ subject to constraints on total $M$ and $N$ above, apply Stirling's formula to the factorials, introduce a Lagrangian multiplier $\gamma(t)$ and obtain

$$
\frac{m_{n}(t)}{M}=\frac{e^{\gamma(t) n-\frac{\epsilon_{n}}{k T}}}{\sum_{n} e^{\gamma(t) n-\frac{\epsilon_{n}}{k T}}}
$$

Phenomenologically, we note that inversion occurs when the energy change for additional pooling requires additional phonon excitation of the solid that exceeds the Debye peak 
discontinuity in phonon density of states of the solid. We thereby assume that the vibrational number domain for pooling is restricted by (units $\hbar=1$ ):

$$
\omega_{D} \geq \epsilon_{1,0}-\epsilon_{n_{\max }, n_{\max }-1},
$$

where $n_{\max }$ is the maximum integer $n$ attainable energetically by a one-quantum transfer from an $n=1$ neighbor by pooling, and so satisfies Eq. (5). The pooling maximum arises because of the discontinuity in the density of states at the Debye frequency $\omega_{D}\left(223 \mathrm{~cm}^{-1}\right.$ where in Eq. (5), $\epsilon_{n, m}=\epsilon_{n}-\epsilon_{m}$, with $\epsilon_{n}$, the vibrational energy of the oscillator, in this case the adsorbed diatomic molecule, given by

$$
\epsilon_{n}=\omega_{e}\left(n+\frac{1}{2}\right)-\omega_{e} \chi_{e}\left(n+\frac{1}{2}\right)^{2} .
$$

Here, $\omega_{e} \chi_{e}=11.5 \mathrm{~cm}^{-1}$ is the anharmonicity and $\omega_{e}=2130$ $\mathrm{cm}^{-1}$, known from CO infrared spectra. In virtue of Eq. (5), we restrict the domain to $\left[0, n_{\max }\right]$.

We rewrite Eq. (4) as

$$
\ln \left(m_{n}\right)+\frac{E_{n}}{k T}=\gamma n+\ln (M)
$$

We note that if $\ln \left(P_{n}\right)+E_{n} / k T$ is a linear function of $n$, then the slope is $\gamma$, the only parameter in our distribution.

We can test Eq. (7) by comparing with kinetic Monte Carlo results on the ms experimental timescale (Ref. 3 gives further details of the calculation). The result is seen in Fig. 1 and is evidence of the usefulness of the theory in the present paper for the constrained distribution of vibrational quanta among the quantum states of the oscillator. The distribution given by Eq. (4) is not exact. Nevertheless, the results demonstrate that it is a useful description of the inverted distribution with its cutoff at $n=10$.

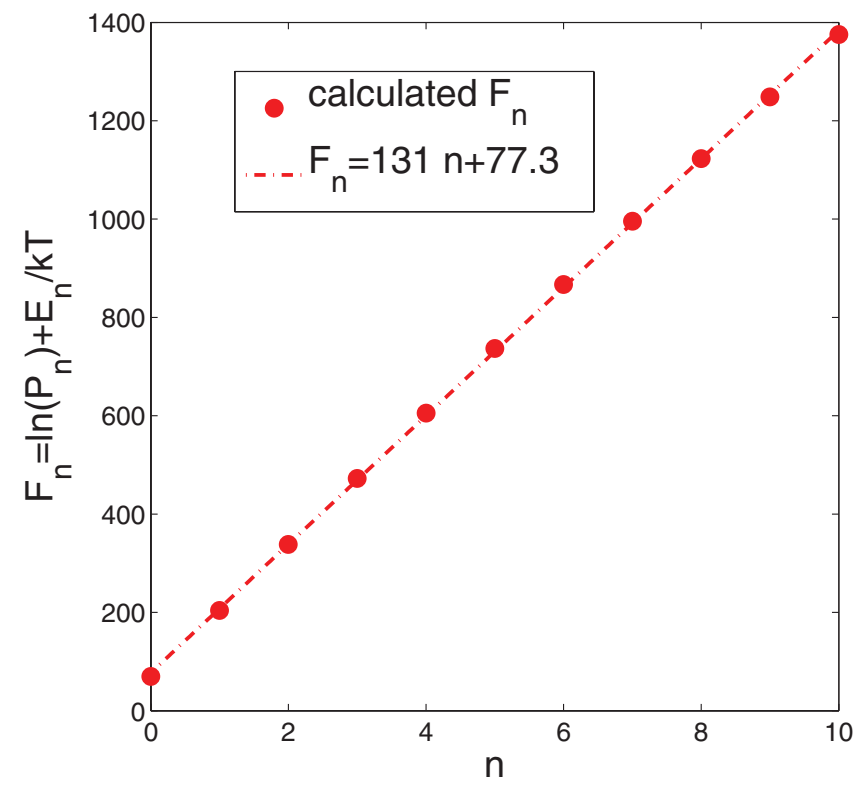

FIG. 1. The fit for the parameter, $\gamma$. The representative pool is derived from monolayer populations, calculated elsewhere by kinetic Monte Carlo, ${ }^{3}$ as the slope of $\ln \left(P_{n}\right)+E_{n} / k T$, vs. $n$, the vibrational state. For the $P_{n}$ calculated near the end of a pulse of the conditions of the previous monolayer experiment, ${ }^{10}$ $\gamma=131$

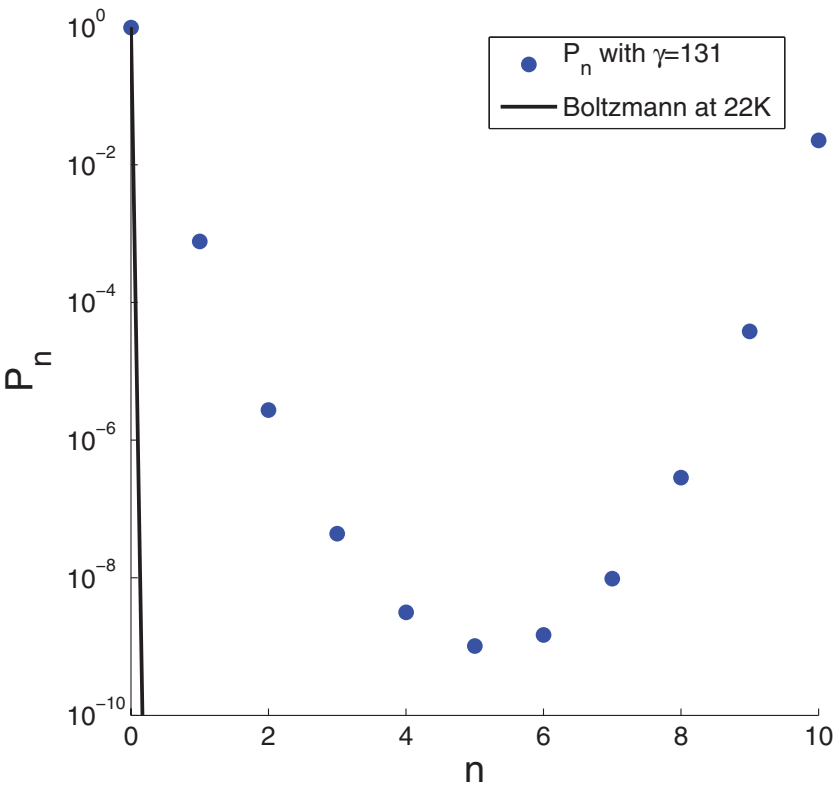

FIG. 2. This figure shows the $P_{n}$ from Eq. (4) compared to the Boltzmann distribution at $22 \mathrm{~K}$ for $\gamma=131$.

The $\gamma$ appearing in Eq. (4) can be evaluated independently from the following:

$$
\frac{N}{M}=\frac{\sum n e^{\gamma n-\frac{\epsilon_{n}}{k T}}}{\sum e^{\gamma n-\frac{\epsilon_{n}}{k T}}} .
$$

A simple way of obtaining $\gamma$ from the value of $N / M$ is to evaluate the right-hand side of this function for varied $\gamma$, and then find the $\gamma$ corresponding to the experimentally known $N / M$. Given a lasing rate of $k_{a b s}=9.0 \times 10^{-4}$, one expects a long term excitation of $N / M=\left(1-\exp \left(-k_{a b s} t\right)\right) /$ $2=0.18 .{ }^{10}$ From this value for $N / M$, we find $\gamma=130$, agreeing to every significant figure with the result derived from kinetic Monte Carlo $P_{n}$, as shown in Fig. 2.

\section{RESULTING DYNAMICS}

The dynamics in the Monte Carlo simulations are quite complex, ${ }^{1-3}$ in containing hundreds of first-order and secondorder reactions, but can be treated as having to an effective single exponential decay when there is a rapid equilibration process among the states $n$ as follows. Consider the average number of quanta in any one site:

$$
N=\sum n m_{n}
$$

In the loss of $N$ vibrational quanta from the pooled surface, the non-radiative excitation of phonons in the solid involves many phonons, and is slow relative to single-quantum pooling equilibration: the latter involves only single excitations, whereas the former involves many such excitations simultaneously. The slow disappearance of quanta in the adsorbate is given by

$$
-\frac{d N}{d t}=\sum \kappa_{n} m_{n}
$$

If there is at each time $t$ a rapid equilibration among the quanta in the adsorbate, then there is a single exponential decay of 
$N, N=N_{0} e^{-\lambda t}$, and $m_{n}=m_{n_{0}} e^{-\lambda t}$, so from Eq. (9) we have $d N / d t=-\lambda \sum n m_{n}$. We note that this temporal dependence of the $m_{n}$, under rapid pooling equilibration, leads to the same, single-exponential, temporal decay of all states equilibrated, with time constant $1 / \lambda$, in contrast to prior expectations for pooling on the $\mathrm{H}: \mathrm{Si}(111)$ surface. ${ }^{8}$

Comparing with Eq. (10), we then have

$$
\lambda=\frac{\sum \kappa_{n} m_{n}}{\sum n m_{n}} .
$$

When applied to the present problem, ${ }^{3}$ this model with the theoretical constrained equilibrium distribution given in Eq. (4) recovers a reasonably close time constant $(3.6 \mathrm{~ms}$ for the present theoretical result of Eq. (11) vs. $4.3 \mathrm{~ms}$ in the full Monte Carlo calculation and experimentally) and single exponential behavior for each state in the pool with the same time constant. We can compare the effective single exponential decay rate with the actual computed results for the monolayer, as in Fig. 3. We note that the actual output of the Monte Carlo code is not a perfect fit to a single exponential with the same exponent at all times, but varies from having slightly shorter to slightly longer effective time-constants throughout the calculation (as in Fig. 3). Nonetheless, when plotted on the observable, total overtone fluorescence scale, $I(t)$ vs. t, as in the computational comparison with experiment, ${ }^{3}$ the difference from single exponential for the monolayer result is barely noticeable, as in Fig. 4.

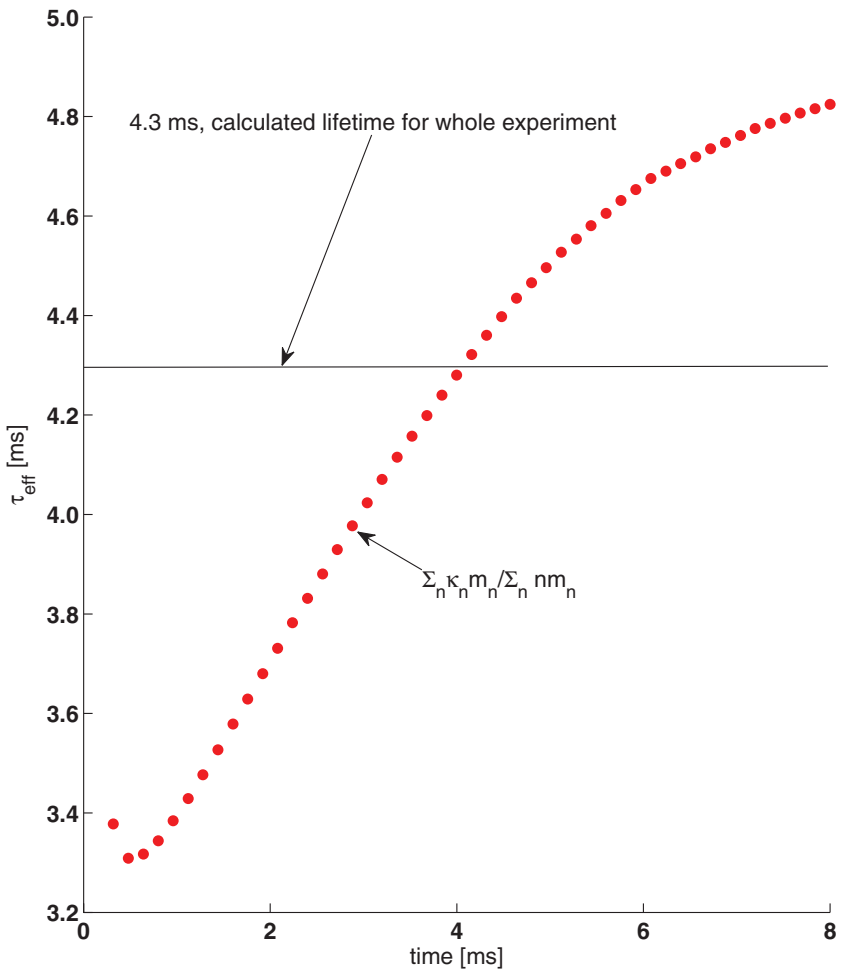

FIG. 3. Calculated $1 / \lambda(t)$ versus time, compared with the single exponential observation. The deviation is small, varying from shorter lived at short times to longer lived at long times. The difference from the best-single-exponential fit for the overtone fluorescence in paper, is negligible on the ms timescale when plotted for the observable directly, as in Ref. 3.

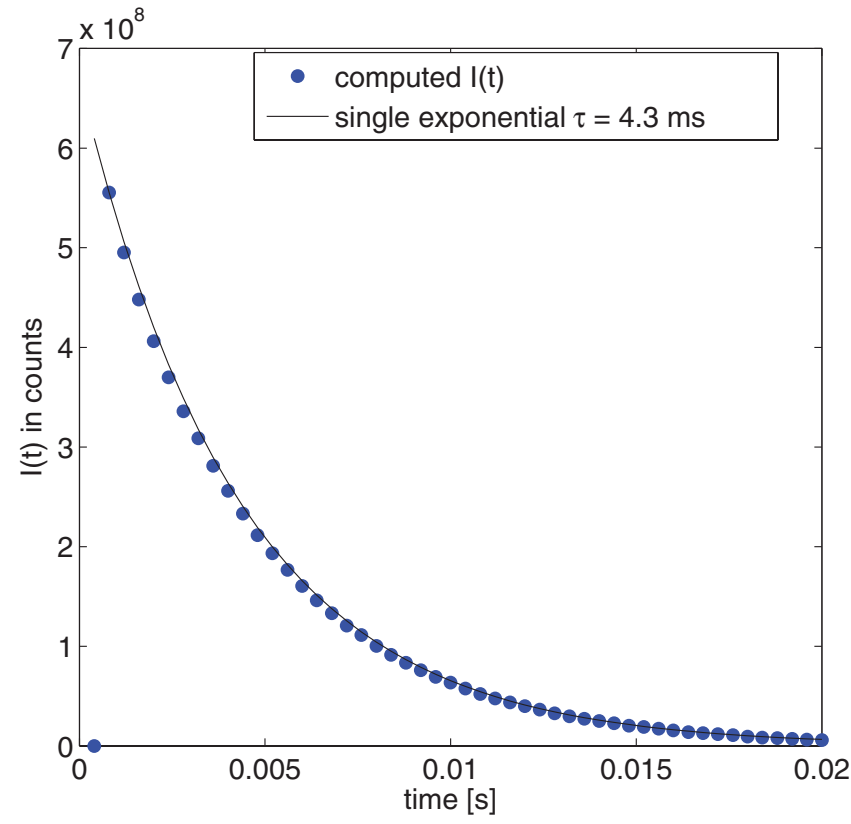

FIG. 4. The total overtone fluorescence decay (circles computed) matches experiment (solid single exponential with $\tau=4.3 \mathrm{~ms}$ ).

\section{DISCUSSION}

The consequences of a novel regime of distribution of the vibrational quanta among the different vibrational states are several-fold. ${ }^{6,7}$ We note the unifying simplicity of application of the model for different surfaces and phases. While our $\mathrm{CO}: \mathrm{NaCl}(100)$ simulations at several laser intensities is a time-consuming calculation, ${ }^{3}$ the simplicity of the present approximate analytical distribution, when valid, allows one to describe readily other results that may occur experimentally.

We note that the single exponential decay for individual states calculated in this work and Refs. 1-3 indicates that a single exponential decay of individual states cannot be taken as evidence against vibrational pooling, as has been suggested for the H:Si(111) surface. ${ }^{8}$ Single exponential decay, even of individual states as observed experimentally (see the discussion preceding Eq. (11)), does not rule out pooling of the vibrational excitation as long as the latter is rapid relative to the decay to the solid, and this point plays a major role when considering fast bimolecular vibrational processes such as singlequantum assisted non-resonant energy transfer. The key to a single exponential is the validity of the approximation of rapid equilibration between the quantum states of $\mathrm{CO}$ molecules on the surface. For example, if a particular state $n^{\prime}$ has a relatively rapid decay rate by energy loss to the solid (still slow relative to the pooling equilibration, but fast relative to all other processes), the other $n$ s rapidly refill the $n^{\prime}$ population, so that all states $n$ decay at the same rate. In summary, rapid equilibration among the states relative to loss of quanta to the solid is the key to understanding the single-exponential decay observed both in the experiment and in the computations.

A comparison of the present derivation $(\mathrm{CO}$ on a solid) with Treanor's ${ }^{6}$ (CO in a gas) is interesting. The $N$ conservation step is crucial in both, and, up to our Debyebased cut-off, the resulting distribution function is of the same 
form for both (Treanor's ${ }^{6}$ equation (4.8) and Eq. (4) of this paper). In our case, there is a restriction of domain of allowed $n$-states in the single-quantum exchange with the solid, whereas in the gas phase the non-resonant transfer was aided by a smooth translational energy distribution. However, another difference, purely technical, rather than physical, is in the minimization of free energy in our derivation, as opposed to an entropy-maximization and a temperature ansatz for $\beta$ in Treanor's. The latter ansatz requires further steps to be made rigorous, namely, a calculation of the energy $E$, entropy $S$, and an introduction of temperature, $1 / T=d S / d E$, to reach a rigorous result, a result obtained simply by a free energy minimization as above.

Of particular interest in the present study is the inverted nature of the distribution, with a maximum at some $n_{\max }=\left\lceil\omega_{D} / 2 \omega_{e} \chi_{e}\right\rceil$ following a period of excitation. We note that this limit is proportional to the Debye cutoff frequency and inversely proportional to the anharmonicity, so $n_{\text {max }}$ for CO:Si(100) would be 20 because $\omega_{D}=448 \mathrm{~cm}^{-1}$ for $\mathrm{Si}(100),{ }^{15}$ assuming the same anharmonicity for $\mathrm{CO}$, while for $\mathrm{H}: \mathrm{Si}(111)$ it would be 5 , since $2 \omega_{e} \chi_{e}=90 \mathrm{~cm}^{-1}$ for that surface, assuming the same $\omega_{D}$ as $\operatorname{Si}(100)$. Additional investigation of these surfaces using kinetic Monte Carlo and treating several experimental results ${ }^{8,13}$ is the topic of forthcoming work. Pooling equilibration, while minimizing the free energy, also conserves the total number of quanta. Because of this constraint, the population tends to lower its energy by occupying the highest vibrational states, and so inversion is thermodynamically allowed, consistent with a constrained equilibrium statistical mechanics. Complete decay, radiatively and non-radiatively, will occur to eventually yield a thermal equilibrium population distribution, largely in the $n=0$ state.

The present approximation is not restricted to phonon relaxation of high frequency vibrations on solids, but is relevant in other situations that conserve $N$, the total number of vibrational quanta, namely, situations where there is rapid singlequantum non-resonant vibrational transfer, faster than dissipative processes. In all cases, there is then superimposed on these rapid exchanges the slow decay of $N$. The theory may be extended to treat isolated molecules in matrix solids, ${ }^{9}$ and multilayers of $\mathrm{CO}$ on $\mathrm{NaCl}(100))^{4,5}$

The validity of the equilibration approximation depends on the absorbed laser intensity. It may be expected to be valid when the intensity is sufficiently high. The argument is as follows: equilibration is valid when the decay rate for loss of quanta to the solid is small relative to the rates of the pooling and depooling processes. Pooling is a second-order process and proportional to the square of the light intensity. If the absorbed intensity is too low, the rate of realized pooling will be too slow and the approximation fails.

Quantitatively, for monolayer $\mathrm{CO}: \mathrm{NaCl}(100)$, where the observed fluorescence relaxation lifetime is $4.3 \mathrm{~ms}$, the calculated rate constant for the $1+9$ to $0+10$ pooling reaction is $5 \times 10^{7} \mathrm{~s}^{-1}$ from the kinetic Monte Carlo calculations. ${ }^{2,3}$ For this case, wee see that the pooling rate constant, $k_{\text {pool }}$ is $2 \times 10^{5}$ times faster than the rate of loss of single quanta to the solid, $\lambda \approx 1 /(4.3 \mathrm{~ms}$ ) (noting again slight variation around this mean lifetime over time as in Fig. 3). If $k_{\text {pool }} P_{1} P_{9} \gg \lambda P_{9}$, we expect the equilibration condition to be satisfied, and so when $P_{1} \gg \lambda /\left(k_{\text {pool }}\right) \approx 5 \times 10^{-6}$. Knowing or estimating the cross-section for the absorption and a lifetime for the loss, from $n=1$ to $n=0$, for quanta to the solid, one can estimate what laser intensity is needed to obtain any $P_{1}$.

In the case of possible vibrational pooling in $\mathrm{H}: \mathrm{Si}(111)$ Sum Frequency Generation (SFG) pump-probe experiments, there is observed single exponential decay of the $n=1$ state. $^{8}$ There was also observed a hot band, ${ }^{13}$ whose observed lifetime is the same as that for recovery of the fundamental at room temperature, $0.9 \mathrm{~ns}$. If the rapid equilibration (fastpooling) approximation holds, then all $0<n \leq n_{\max }$ should have the same single-exponential rate of relaxation, identical to the rate of $n=0$ recovery. If there were no equilibration, the lifetimes for $n=1$ and $n=2$ would be quite different. For example, computations ${ }^{12}$ for a Bloch-Redfield dynamics gave an intrinsic lifetime for the $n=2$ state of $0.13 \mathrm{~ns}$, and for the $n=1$ state, 0.9 ns. From previous calculations of the pooling rate constant, ${ }^{11}$ the rate constant for the $1+1$ to $0+2$ pooling reaction can be as high as $\approx 2 \times 10^{8} \mathrm{~s}^{-1}$ on $\mathrm{H}: \mathrm{Si}(111)$ under some experimental conditions. ${ }^{8,13}$ Based on the trends in pooling rate constants for $\mathrm{CO}: \mathrm{NaCl}(100),{ }^{3}$ we expect the pooling rate constant for the $1+n_{\max }-1$ to $0+n_{\max }$ pooling reaction, $k_{\text {pool }}^{\mathrm{SiH}}$, to be $7 \times 10^{9} \mathrm{~s}^{-1}$. In this case, the calculated pooling rate constant is $\approx 10$ times the observed rate of loss of single quanta to the solid $\left(\lambda^{\mathrm{SiH}} \approx 1 / 0.9 \mathrm{~ns}=1 \times 10^{9} \mathrm{~s}^{-1}\right)$, and $P_{1} \gg 0.1$ would meet the equilibrium condition.

One can also examine the spectrally integrated SFG intensity in the previous hot band pump-probe experiment for evidence of pooling on $\mathrm{H}: \mathrm{Si}(111) .{ }^{13}$ We plan to discuss these and other issues ${ }^{14}$ for the $\mathrm{H}: \mathrm{Si}(111)$ system in a later publication.

\section{CONCLUSIONS}

In the present theory, a simple distribution is derived by a free energy minimization during vibrational-quantaconserving pooling equilibration on solids. In particular, the following experimentally testable predictions are made:

(1) Statistical behavior is expected in vibrational equilibria, subject to the constraint of a slowly decaying number of quanta $N(t)$ when the vibrational equilibration is fast relative to all radiative and non-radiative processes. This behavior can be described by the temperature and Debye frequency of the solid along with the anharmonicity and fundamental frequency of the high frequency vibration.

(2) All vibrational populations on surfaces in this model are described by restricting quantum state $n$ to the domain $\left[0,\left\lceil\omega_{D} / 2 \omega_{e} \chi_{e}\right\rceil\right]$. The vibrational pools are coupled by all resonances and near-resonances consistent with the preservation of the number of quanta.

(3) Temporal single-exponential decay cannot be taken as evidence against vibrational pooling despite the bimolecular rate constant behavior of individual rates and the unimolecular dependence of other steps. Indeed, single exponential decay is the expected result if pooling equilibration is faster than all decay processes. 


\section{ACKNOWLEDGMENTS}

We thank the ARO, ONR, and NSF for the support of this research, Dr. Nima Ghaderi for useful discussions, and the reviewers for their helpful comments.

${ }^{1}$ S. A. Corcelli and J. C. Tully, J. Phys. Chem. A 106, 10849 (2002).

${ }^{2}$ S. A. Corcelli and J. C. Tully, J. Chem. Phys. 116, 8079 (2002).

${ }^{3}$ E. T. D. Boney and R. A. Marcus, "On the infrared fluorescence of monolayer ${ }^{13} \mathrm{CO}: \mathrm{NaCl}(100)$," J. Chem. Phys. (submitted).

${ }^{4}$ H.-C. Chang and G. E. Ewing, J. Phys. Chem. 94, 7635 (1990).

${ }^{5}$ H.-C. Chang, C. Noda, and G. E. Ewing, J. Vac. Sci. Technol. A 8, 2644 (1990).
${ }^{6}$ C. E. Treanor, J. W. Rich, and R. G. Rehm, J. Chem. Phys. 48, 1798 (1968).

${ }^{7}$ D. McQuarrie, Statistical Mechanics (University Science Books, 2000).

${ }^{8}$ P. Guyot-Sionnest, P. Dumas, and Y. J. Chabal, J. Electron Spectrosc. Relat. Phenom. 54/55, 27 (1990).

${ }^{9}$ I. H. Bachir, R. Charneau, and H. Dubost, Chem. Phys. 177, 675 (1993).

${ }^{10}$ H.-C. Chang and G. E. Ewing, Phys. Rev. Lett. 65, 2125 (1990).

${ }^{11}$ J. Ma, E. Wang, Z. Zhang, and B. Wu, Phys. Rev. B 78, 125303 (2008).

${ }^{12}$ H. Gai and G. A. Voth, J. Chem. Phys. 99, 740 (1993).

${ }^{13}$ P. Guyot-Sionnest, Phys. Rev. Lett. 67, 2323 (1991).

${ }^{14}$ Z. Liu, L. C. Feldman, N. H. Tolk, Z. Zhang, and P. I. Cohen, Science 312, 1024 (2006).

${ }^{15}$ C. Kittel, Introduction to Solid State Physics, 8th ed. (John Wiley \& Sons, 2004). 